\title{
Surprise! students don't do special-case analysis when unaware of it
}

\author{
MacKenzie Lenz, ${ }^{1}$ Paul J. Emigh, ${ }^{1}$ and Elizabeth Gire ${ }^{1}$ \\ ${ }^{1}$ Department of Physics, Oregon State University, Corvallis, OR 97331
}

\begin{abstract}
Special-case analysis - setting parameters to special values and checking that the answer is consistent with previously known results or physical intuitions - is a common strategy for reflecting on the correctness and meaning of answers to physics problems. We interviewed eleven calculus-based introductory physics students to learn about their use of such reflection strategies. Six of the students were enrolled in a reformed course where students were prompted to reflect on their homework solutions. Checking special cases was specifically suggested in the homework instructions as a reflection strategy. Five students were in a different course that did not prompt reflection on homework. During the interviews, none of the students performed a special-case analysis or were familiar with the strategy. We suggest that students need explicit instruction on how to do a special-case analysis if they are expected to perform it while reflecting on answers to physics problems.
\end{abstract}

\section{INTRODUCTION}

Physics sensemaking - seeking meaning or coherence between representations of physics knowledge - is an important aspect of doing physics. Sensemaking is a large part of physics problem solving. To teach problem solving, instructors often use rubrics or frameworks, many of which contain a reasonableness check or evaluative sensemaking step [1-4].

Special Case Analysis (SCA) is one evaluative sensemaking strategy. For example, one might make sense of a symbolic equation by setting a parameter to a special value and checking for consistency with previously known results or physical intuitions. SCA includes limiting-case analysis or checking extremes. Instruction in SCA can improve students' ability to answer multiple-choice questions correctly [5]. However, even more advanced physics students tend not to use SCA spontaneously [6]. Also, minimal work has been done to analyze how students make sense of answers they did not generate themselves (i.e., independent of problem solving).

In this exploratory study, we gave introductory physics students a symbolic answer to a physics problem and asked them to make a judgment about whether or not the answer is reasonable without explicitly solving the problem. We chose a problem and answer where SCA is particularly useful for making a judgment. In this paper, we identify and illustrate the sensemaking students did in order to make their judgment, particularly their knowledge and performance of SCA.

\section{INSTRUCTIONAL CONTEXT}

The research subjects were students enrolled in the first quarter of the calculus-based introductory physics sequence at Oregon State University, a large, public, research-intensive institution. The course is offered in two different formats: Traditional and Studio.

The Traditional format met for three one-hour lectures taught by one instructor and one three-hour lab each week. The homework and exams did not prompt or require reflection (evaluative sensemaking). Students were encouraged to solve problems symbolically before plugging in numbers.
TABLE I. Excerpt from the required solution format and grading rubric to which Studio students had access.

\begin{tabular}{l}
\hline Required Solution Format: Reflect. Is the answer \\
reasonable? Does it make physical sense? \\
- Evaluate the result. Are the units correct? \\
swer reasonable? Is the an- \\
Does the answer make sense in limiting cases? Does \\
the answer make physical sense? Include a written \\
explanation for why the answer makes sense and what it \\
implies about the physical system.
\end{tabular}

Description of a Full Credit Answer on Grading Rubric: A clear and complete explanation is given for why the result makes sense (or does not make sense if the incorrect answer was reached), and what it tells us about the physics of the situation.

The Studio format met for two one-hour lectures, one two-hour studio, and one two-hour lab each week. Each lecture section was taught by a different instructor and therefore the Studio had less consistency across sections than the Traditional format. Studio consisted of small-group problem-solving activities that were the same across all three instructors. Although the instructors wrote different exams, all used the same homework and rubric that explicitly included a prompt for reflection. Most homework problems asked for numerical answers but students were encouraged to solve the problem symbolically first. Table I shows the reflection portion of the solution format and grading rubric. The sentence in gray is a prompt for students to do SCA and is the inspiration behind the interview question analyzed here.

Three instructors were asked about their teaching practices around SCA (one of the Studio instructors could not be reached). These questions were asked as a follow up to the course and collection of the unexpected results discussed in section IV. All three instructors said they demonstrated SCA in class but did not teach SCA explicitly. For example, when solving a problem in class an instructor may have said or, more rarely, written down an example of SCA. This demonstration is a common way of teaching evaluation strategies in physics courses [7]. 
Interviewer: Without solving the problem, reflect upon the answer given to see if it makes sense or not.

Written: "You placed your physics book on a table and it starts to slide because your table is uneven, yikes! Since you have been studying physics all day you can't help but wonder at the acceleration of your textbook. If you look up the average coefficient of friction for a wood table and measure the angle of incline what expression can you use to determine the acceleration of your book?"

$$
a_{x}=g\left(\cos (\theta)-\mu_{k} \sin (\theta)\right)
$$

FIG. 1. This prompt was designed to investigate each student's ability to identify incorrect trig functions; we anticipated they would use special-case analysis (SCA).

All three instructors stated that SCA is an important skill for their students (mostly engineering majors) and believed their students capable of performing SCA at this level. However, they did not expect to see SCA on students' written work during the first quarter, but rather expected to see this skill develop and become more prevalent on written work in the second and third quarters.

\section{METHODS}

As part of a larger study to understand students' use of evaluation strategies, we performed semi-structured interviews with 6 Studio students (S1-S6) and 5 Traditional students (T1-T5). These hour-long interviews had a think-aloud protocol [8] with three parts: one or two problems to be solved, one or two problems with an answer given to be analyzed, and a discussion about the course and evaluation strategies. One extra question was given to the Traditional students. This question was added as a warm-up after the initial (Studio) interviewees found the other problem to be complicated, difficult, and discouraging. The new question is a frictionless variant of the prompt in Fig. 1.

In this manuscript, we focus on a question given in the second part (see Fig. 1). This prompt was intended to explore what strategies students use to evaluate a symbolic answer that is incorrect but dimensionally consistent. In particular, this prompt can be verified as incorrect by checking the value of $a_{x}$ in either the special case of $\theta=0$ or $\theta=\pi / 2$. Alternatively, the expression is correct if using the complementary angle. The interviewer aided the students in drawing the correct angle to ensure that no one used an incorrect diagram. The interviewer interrupted the student if the student began to solve the problem symbolically rather than evaluate Eq. 1.

In the last part of the interviews, students were asked if they knew or used SCA. The interviewer used many terms to describe SCA, including "limiting-case analysis" and "taking to extremes," to help students access any knowledge related to SCA.

All interviews were audio- and video-recorded and then transcribed (with hand gestures). Students worked on multiple large whiteboards. After students completed each problem, the whiteboard was removed (so students could not erase their work) and later photographed.

A thematic analysis was used to analyze the data [9].
The transcripts were read multiple times by one researcher to identify relevant themes. These themes were then discussed among all authors. After discussion one researcher went through the transcripts again to ensure all instances of the identified themes were gathered.

We also performed semi-structured interviews with the Traditional instructor and two of the Studio instructors (the third Studio instructor could not be reached). These interviews were approximately 30 minutes long and occurred as a follow-up to the student interviews. They focused on how SCA was taught and each instructor's expectations for student use. Results from these interviews were discussed in section II.

\section{RESULTS}

Although the given prompt is amenable to SCA (and it was our intent that students use this method to make sense of the given symbolic solution), no students preformed SCA. The claim that Eq. 1 is incorrect was reached by $4 / 11$ students. Only T3 gave correct reasoning for this answer based on their prior knowledge.

Despite no students using SCA, there were many other strategies and approaches that students used to make sense of Eq. 1 without explicitly solving the given problem. Most of these approaches did not prove fruitful in identifying Eq. 1 as incorrect. However, some did appear to help students understand the given situation. Identifying such approaches helps us better understand how students were seeking meaning or coherence between representations of physics knowledge.

In this section, we first discuss the different approaches that students took to make sense of Eq. 1. We then follow these up with what students knew about SCA from the discussion section of our interviews.

\section{A. Most students drew free-body diagrams}

All but one student (T5) drew a free-body diagram (FBD). Students drew a FBD primarily at the onset of their analysis and some added to or edited their diagram as they continued their analysis. Drawing a diagram appeared to be a habitual practice for students. For example, T4 expressed that drawing a diagram is something they always do when solving physics problems.

\section{Well if you draw it out. I can't help it. (T4)}

The level of detail in the FBDs varied. S3 drew a simplified diagram consisting of arrows but no labels of force vectors or axes (see Fig. 2 left). On the other hand, T2 drew a more expert-like FBD consisting of labeled force vectors and axes (see Fig. 2 middle). T2 also included the relevant angle, drew and labeled the relevant forces, and indicated tilted axes using both words and hand gestures.

Drawing FBDs was a useful sensemaking strategy for students: angles lead to thinking about geometric relations, arrows/vectors lead to identifying relevant forces, and axes helped students orient Eq. 1 along the table. However, the FBDs alone were not enough for students to make a judgment about Eq. 1 but instead served as a precursor to further discussion. 

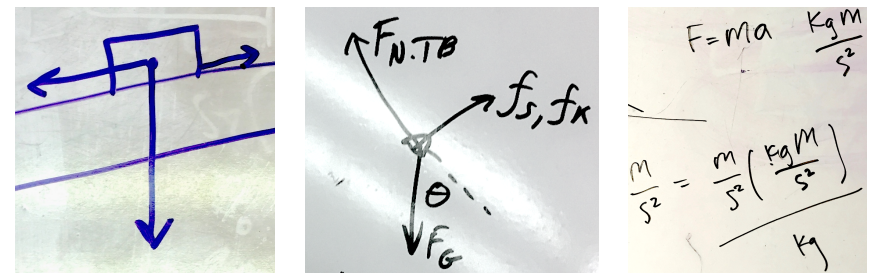

FIG. 2. Three examples of student work: (left) S3 drew a simple free body; (middle) T2 drew a more sophisticated free body diagram; (right) S2 uses units to check Eq. 1.

\section{B. Many students talked about friction}

Friction was discussed by $7 / 11$ students. Most $(5 / 7)$ gave an equation for calculating the friction force (T3, $\mathrm{T} 4, \mathrm{~S} 4, \mathrm{~S} 5$, \& S6), as seen in the quote below from S6.

Force of friction is going to be $-\mu_{k}$ times $g$. (S6)

These five students identified that the $\mu_{k} \sin (\theta)$ term in Eq. 1 represents the acceleration due to friction.

This has the kinetic friction force, this is the kinetic friction acceleration [points at $\mu_{k} \sin (\theta)$ term]. (S4)

Static friction was mentioned by $3 / 7$ of these students: T2, T4, and S4. Student T2 labeled both static friction and kinetic friction on their FBD, as seen in the middle of Fig. 2 where both friction forces are assigned to one arrow. It is unclear if $\mathrm{T} 2$ knew which friction was acting on the book as they only discussed friction once. Students $\mathrm{S} 4$ and T4 stated that static friction does not need to be included in the problem, as demonstrated below.

Static friction isn't taken into place ... because it's so small. (S4)

Mass was related to friction only by S2, who reasoned that Eq. 1 is incorrect because friction depends on mass and therefore $a$ should also depend on mass, as below.

It's just this part [points at $F=$ ma] but you need the mass because depending on the mass that will change how much the friction affects it [the acceleration]. (S2)

Considering the effect of friction helped $3 / 7$ students eliminate static friction as a relevant force and $5 / 7$ to identify the friction term in Eq. 1. Identifying terms is a sensemaking strategy analogous to examining functional dependence. One student incorrectly reasoned that Eq. 1 is incorrect by considering friction. Overall, this approach helped students make sense of the given problem situation and continue evaluating Eq. 1.

\section{Many students were confused by the trigonometric functions}

About half the students (6/11) showed confusion about trig functions (S2, S4, S5, T2, T4, \& T5). For example, T5 expressed the following discomfort with trig functions.

My brain is pretty hazy about sines even when I write problems out so looking at the symbolic answer it's just like 'oh ya I'll take your word for it'. (T5)
Such confusion was frequent when students tried to state whether or not Eq. 1 is correct, as in the example below.

I would have to actually look at the angle to make sure that it's right because I hate these angles. (S4)

Ultimately S4 did not see anything explicitly wrong with Eq. 1 but concluded, looking at the trig functions, that it is incorrect because "somehow it sits uneasy with me."

Two students (S4 \& T4) identified Eq. 1 as incorrect based on their analysis of the trig functions, though their reasoning was incorrect. S4's reasoning was that Eq. 1 seemed wrong with no further explanation (see above) and $\mathrm{T} 4$ only ruled out the $\sin (\theta)$ term but not the $\cos (\theta)$ term. The other $4 / 6$ students expressed confusion about the trig functions. These 6 students did not display any sensemaking strategies allowing them to overcome their confusion about trig functions. However, they did tend to show a high level of metacognition about this aspect of the problem. Trig functions therefore seem to be an area of contention for these students that future instruction, including a focus on SCA, might be able to help.

\section{Some students checked units}

Units were checked by 4/11 students, all from the Studio course (S1, S2, S5, \& S6). An example is below.

$g$ is by definition acceleration, $\mu$ is unit-less, cosine and sine are unit-less so that [pointing at Eq. 1] is an acceleration. (S6)

One student performed a unit check (see Fig. 2 right), incorrectly identifying the trig functions as having units of $\mathrm{kg} \mathrm{m} / \mathrm{s}^{2}$, which led to incorrect unit analysis of Eq. 1 .

Checking units was performed at various times: S1 and $\mathrm{S} 2$ at the end, S5 in the middle, and S6 at the beginning of students' evaluations. The units of Eq. 1 were identified as correct by $3 / 4$ students. This identification lead S1 to stop and say Eq. 1 is correct and S5 and S6 to attempt other strategies. Unit checking helped students identify what is correct about Eq. 1 and lead 2/11 students to explore other aspects of the equation, making further connections among physics knowledge.

\section{E. Some students discussed mass}

The mass of the book was explicitly addressed by $5 / 11$ students (S1, S2, S5, T3, \& T1). It was recognized by $4 / 5$ that Eq. 1 should be independent of mass.

You can divide your mass out because the mass is the same throughout the entire problem so mass goes away which I agree there shouldn't be any mass in here (T1)

Identification and/or expectation that Eq. 1 is independent of mass was done with a unit analysis or through identification of what the symbols in Eq. 1 represent.

S2 stated that Eq. 1 should be divided by mass, as shown in the unit analysis on the right of Fig. 2 (see section IV B for more detail). S2 used this incorrect reasoning to claim that Eq. 1 is incorrect. By identifying what Eq. 1 is not dependent on, students were able to rule out quantities as affecting the acceleration, another example of functional dependence. 


\section{F. Few students referenced prior knowledge}

T3 \& T5 mentioned the warm-up question that was added to the protocol. This added problem may have prompted them to address their prior knowledge. T3 used correct reasoning to identify that Eq. 1 is incorrect by referencing the added problem both in words (see the quote below) and by pointing at the whiteboard where they had previously solved the problem.

Like I did back on problem 1 ... without friction it would be $g \sin \theta$. (T3)

In contrast, student $\mathrm{T} 5$ referred to the added problem but was not able to use it to make a claim about Eq. 1 because they remained unsure of the trig functions. However, they still found this problem helpful:

It's always good to have schema of prior knowledge to link things to. (T5)

Using prior knowledge to make sense of a situation or an answer to a problem is another expert-like sensemaking skill. This strategy was the only one used as correct reasoning to identify Eq. 1 as incorrect.

\section{G. Students were unfamiliar with SCA}

During the last part of the interview, 5/11 students described a vague familiarity with $\mathrm{SCA}(\mathrm{S} 1, \mathrm{~S} 2, \mathrm{~S} 6, \mathrm{~T} 3$, \& T4). Students S6 and T4 recalled what SCA is just by the interviewer asking if they remember it. Both expressed that SCA was not an important approach in their physics course and that they learned it outside the course.

Not in this class but I have heard the term before ... my vector calc [instructor] has [discussed SCA]. (T4)

Students S1, S2, and T3 only recalled what SCA is after being shown an example. They recognized it from demonstrations in lecture but never knew what to call it.

He [the instructor] has done that in class actually but he hasn't referred to what the name is. (S1)

The other 6/11 students did not know what SCA was when asked or demonstrated. Two students stated SCA would be helpful and wished they had learned it earlier.

Students confirmed that SCA was taught in their course through demonstration. Students who were familiar with SCA did not see how it could be of use in their physics course. Overall students expressed that SCA was useful when shown how to apply it to Eq. 1.

\section{CONCLUSION}

None of the students we interviewed used Special Case Analysis to evaluate the correctness of a given solution, a result that we did not anticipate. Half the students knew or recognized SCA after it was demonstrated to them. Even though the other students did not appear to be familiar with SCA, they did believe it would have been helpful. This finding indicates that teaching SCA in introductory physics by demonstration only is not enough for students to know how or when to use SCA. Prompting SCA on the homework rubric was also not effective at getting students to use this strategy, possibly due to students' lack of familiarity. Students' statements that SCA would be useful suggests that they might use it given more explicit instruction about how to perform it.

Our preliminary research intended to investigate SCA concluded that students did not know SCA but instead had a variety of ways of making sense of a given equation. Drawing diagrams, checking units, investigating functional dependence (mass and friction), metacognition about trig functions, and prior knowledge all helped students make connections and understand the problem situation [10]. Prior knowledge even helped one student, T3, correctly identify Eq. 1 as incorrect. Three other students used incorrect reasoning to make the same claim. Clearly, these students have many sensemaking tools at their disposal, some that approach expert-like behavior. Encouraging introductory students to see the value in their own sensemaking is therefore likely to promote student learning and engagement in physics.

For the particular problem we gave, the error was in the trig functions, an aspect that many students expressed discomfort with and for which SCA is helpful. Other strategies students used would have helped identify errors with different aspects of the solution. Given the students' discomfort with trig functions, and the potential for SCA to identify incorrect trig functions, we encourage instructors to make SCA an instructional priority.

\section{ACKNOWLEDGMENTS}

We would like to thank the OSUPER group for their help in this study. We would also like to thank the instructors and students who participated in this study.
[1] M. D. C. et al., in PERC Proceedings (2012).

[2] D. DeMuth, "A logical problem solving strategy,".

[3] A. A. et al., Teaching and Teacher Education 63 (2017).

[4] N. B. Hill, Physics Teacher 54, 291 (2016).

[5] A. R. Warren, Phys. Rev. ST Phys. Educ. Res. 6 (2010).

[6] M. E. Loverude, in PERC Proceedings (2017).
[7] M. Lenz and E. Gire, in PERC Proceedings (2016).

[8] K. Ericsson and H. Simon, Psychological Review 87 (1980).

[9] J. W. Creswell, Research Design: Qualitative, Quantitative, and Mixed Methods Approaches (SAGE Pub., 2013).

[10] K. T. H. et al., in PERC Proceedings (2017). 